\title{
Effects of Extreme High Temperatures on \\ Proliferation, Cell Cycle, Cell Differentiation and ROS of Adipose-Derived Mesenchymal Stromal Cells
}

\section{Yuanhui Gao}

Haikou Municipal People's Hospital and Xiangya Medical College Affiliated Hospital: Haikou Municipal People's Hospital and Central South University Xiangya Medical College Affiliated Hospital

\section{Hui Cao}

Haikou Municipal People's Hospital and Central South University Xiangya Medical College Affiliated Hospital

\section{Shunlan Wang}

Haikou Municipal People's Hospital and Central South University Xiangya Medical College Affiliated Hospital

\section{Linlin Zheng}

Haikou Municipal People's Hospital and Central South University Xiangya Medical College Affiliated Hospital

\section{Haowei He}

Haikou Municipal People's Hospital and Central South University Xiangya Medical College Affiliated Hospital

\section{Zhenyu Nie}

Haikou Municipal People's Hospital and Central South University Xiangya Medical College Affiliated Hospital

\section{Mei Chen}

Haikou Municipal People's Hospital and Central South University Xiangya Medical College Affiliated Hospital

\section{Rong Jiang}

Fujian Medical University

\section{Denggao Huang ( $\nabla$ hdg_qx@163.com )}

Haikou Municipal People's Hospital and Xiangya Medical College Affiliated Hospital: Haikou Municipal People's Hospital and Central South University Xiangya Medical College Affiliated Hospital https://orcid.org/0000-0002-9927-1622

\section{Shufang Zhang}

Haikou Municipal People's Hospital and Central South University Xiangya Medical College Affiliated Hospital 
Research

Keywords: high temperature, adipose-derived mesenchymal stromal cells, proliferation, cell cycle, ROS

Posted Date: August 9th, 2021

DOI: https://doi.org/10.21203/rs.3.rs-764300/v1

License: (c) (i) This work is licensed under a Creative Commons Attribution 4.0 International License. Read Full License 


\section{Effects of extreme high temperatures on proliferation, cell cycle, cell}

\section{differentiation and ROS of adipose-derived mesenchymal stromal} cells

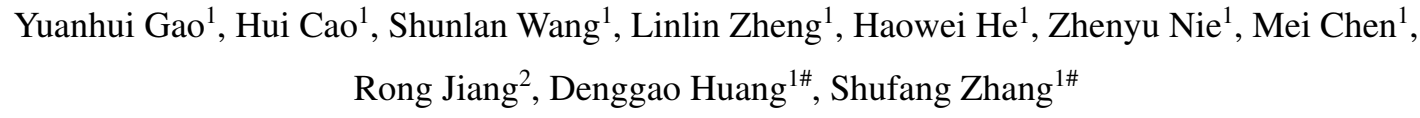

\section{Abstract}

Backgrounds: Global warming has led to extreme temperatures in different latitudinal regions, resulting in the extinction of a large number of species. This study focuses on the effects of extreme high temperatures on cell proliferation, cell cycle, cell differentiation and mitochondria activity in human adipose-derived mesenchymal stromal cells (hADSCs).

Methods: hADSCs were divided into three groups and incubated in $37^{\circ} \mathrm{C}, 39^{\circ} \mathrm{C}$ and $40^{\circ} \mathrm{C}$ environment for 5 hours of exposure each day, and then to $37^{\circ} \mathrm{C}$ circumstances for further incubation. Cell surface markers, cell cycle, cell proliferation activity, mitochondrial activity and cell polarization were detected and analyzed by flow cytometry, CCK-8 assay, ROS and JC- 1 staining respectively on the $1^{\text {st }}$ and $3^{\text {rd }}$ day of cell culture; osteogenic and adipogenic differentiation ability of hASCs was analyzed by staining after 21 days of osteogenic and adipogenic differentiation induction culture.

Results: The results of this study showed that hASCs grown under high temperature conditions had restricted growth activity, blocked S and G2 phases of the cell cycle, reduced cytokinesis and impaired mitochondrial activity, while their osteogenic differentiation ability and membrane potential depolarization were enhanced.

Conclusions: hADSCs were subjected to high temperature stimulation with restricted 
growth activity, reduced cell division, impaired mitochondrial activity, significant cell depolarization and enhanced osteogenic differentiation, and these results were closely related to the pathogenic mechanisms of skin aging and heat stroke due to outdoor sun exposure.

Keywords: high temperature; adipose-derived mesenchymal stromal cells; proliferation; cell cycle; ROS

\section{Introduction}

Recently, global warming has led to extreme temperatures in different latitudinal regions. Some studies have reported that high temperatures from temperate and tropical regions may result in wildlife experiencing a dramatic increase in disease risk ${ }^{[1]}$. As anthropogenic climate change continues to worsen, the risks to biodiversity will increase over time, and future projections suggest that a potentially catastrophic decline in global biodiversity is imminent ${ }^{[2]}$, and these studies suggest that high temperature environments pose a serious threat to both animals and people.

Previous reports have shown that high temperature radiation can cause skin aging and slow down lipid metabolism. Ken Kobayashi et al ${ }^{[3]}$ showed that lactating mice, under moderately high temperature conditions at $39^{\circ} \mathrm{C}$, induced higher lactation capacity of mammary epithelial cells (MECs) through control of STAT5 and STAT3 signaling. In contrast, prolonged exposure to $41^{\circ} \mathrm{C}$ gave rise to a decrease in milk production capacity through inactivation of STAT5 and a decrease in the total number of MECs. It has also been shown that when rats are exposed to high temperature $\left(50^{\circ} \mathrm{C}\right)$, lipolysis in adipose tissue is inhibited due to their high body temperature, while intravascular lipolysis is activated ${ }^{[4]}$. Therefore, understanding the response of human cells to high temperature environment can help us to make positive responses to future environmental changes.

With climate change, the increasing incidence of heat-related deaths has been a frequent concern. The 2019 Global Burden of Disease, Injury, and Risk Factors Study demonstrates the non-optimal temperature as one of the top 10 causes of death globally. Globally, 5 million deaths from 2000 to 2019 are associated with abnormal temperatures ${ }^{[6]}$. For example, a common illness, heat stroke (HS) is a life- threatening disease defined as exposure to excessive hyperthermia at core temperatures above $40^{\circ} \mathrm{C}$ and resulting in a systemic inflammatory response syndrome ${ }^{[7]}$. HS occurs 
when multiple tissues and organs are damaged, allowing the secretion of pro-inflammatory cytokines including tumor necrosis factor- $\alpha$ (TNF- $\alpha$ ) and interleukin-6 (IL-6), and ultimately systemic inflammation ${ }^{[8]}$. However, the specific pathogenic mechanisms need to be further investigated.

Adipose tissue is a highly metabolically active endocrine organ, which plays an important role in energy storage, energy balance and metabolic regulation ${ }^{[9]}$. However, many statistics in recent years have shown that body temperature in healthy adults is gradually decreasing ${ }^{[10-11]}$, suggesting that some changes in adipose tissue in the body are occurring with environmental changes or induced. Therefore, studying the changes in cellular properties in adipose tissue in abnormal environments will help us better understand the effects of environmental changes on the human body. Currently, there are fewer reports on the effects of high temperature environments on adipocytes. The aim of this experiment is to analyze the changes in cell morphology, cell phenotype, cell proliferation, cell differentiation, cell cycle, ROS and membrane potential depolarization of hADSCs at $37^{\circ} \mathrm{C}-40^{\circ} \mathrm{C}$. The purpose of this study was to analyze the effect of high temperature environment on the biological properties of hADSCs.

\section{Materials and Methods}

\subsection{Materials}

Human adipose-derived mesenchymal stromal cells (\#HUXMD-01001) and human adipose-derived mesenchymal stromal cell medium (\#HUXMD-90031) were purchased from Cyagen Biosciences (Guangzhou). Dulbecco's Phosphate Buffer Solution (D-PBS, \#8119217) and Trypsin (\#2177715) purchased from Gibco; Antibodies of CD105-PE (\#560839), CD73-PE (\#550257), CD34-PE (\#348057), CD90-PE (\#561970), CD45-PE (\#557059) purchased from Becton, Dickinson and Company (Guangzhou, China). Cell Counting Kit-8 kit (\#SA616) was purchased from Dojindo; ROS fluorescent probe Dihydroethidium-Hydroethidine (DHE, \#D1008) and Mitochondrial membrane-potential dye (JC-1, \#J4001) were purchased from US EVERBRIGHT, Inc.

\subsection{Methods}

\subsubsection{Cell culture and temperature exposure experiments}

To understood the effect of different temperatures on hADSCs cells. For the 
experiment, Passage 4(P4) of hADSCs cells were adjusted to the appropriate cell density and inoculated in 6-well plates. The cells were incubated in different temperature $\left(37^{\circ} \mathrm{C}, 39^{\circ} \mathrm{C}, 40^{\circ} \mathrm{C}\right)$ incubators for $5 \mathrm{~h}$ every day, and the cells were uniformly placed in the same temperature incubator at $37^{\circ} \mathrm{C}$ after the end of stimulation, then the cell morphology was then observed and photographed. The duration of continuous treatment ranged from 1-28d depending on the design of the different experiments.

\subsubsection{Cell surface marker determination assay}

The cells treated with different temperatures were used to determine the expression of hADSCs surface markers CD105-PE, CD90-PE, CD73-PE, CD34-PE and CD45-PE by flow cytometry. The cells were first digested with $0.25 \%$ trypsin and collected in suspension, the cell density was adjusted $\left(1 \times 10^{5}\right.$ cells $\left./ \mathrm{mL}\right)$ and washed twice with Dulbecco's Phosphate Buffer Solution (D-PBS); the cells were then resuspended in D-PBS, centrifuged at $3000 \mathrm{rpm}$ for $5 \mathrm{~min}$, the supernatant was discarded, the appropriate amount of fluorescently labeled monoclonal antibody was added and incubated for $30 \mathrm{~min}$ at room temperature and protected from light. The cells were resuspended with $300 \mu \mathrm{L}$ D-PBS and analyzed by BD FACSDIVA software, and the surface antigen expression was expressed as a percentage $(\%)$.

\subsubsection{Cell Counting Kit-8 (CCK-8) assay}

When the stimulated cells reach the time point of the assay, aspirate all the medium and add $200 \mu \mathrm{L}$ of fresh medium and $20 \mu \mathrm{L}$ of CCK-8 reagent (10\%), then incubate in the incubator for 1 to $4 \mathrm{~h}$. At the time point, $100 \mu \mathrm{L}$ of supernatant medium was taken into a 96-well plate and the optical density (OD) was read at 450 $\mathrm{nm}$ using an enzyme marker.

\subsubsection{Cell cycle assay}

The P4 cells were adjusted to $10^{6}$ cells per tube and centrifuged at $250 \mathrm{~g}$ for $5 \mathrm{~min}$. The supernatant was discarded, and the cells were fixed in $70 \%$ ethanol at $4^{\circ} \mathrm{C}$ for more than $2 \mathrm{~h}$ and centrifuged at $250 \mathrm{~g}$ for $5 \mathrm{~min}$. $100 \mu \mathrm{L}$ of RNaseA was added to the supernatant for $30 \mathrm{~min}$ in a water bath at $37^{\circ} \mathrm{C} .400 \mu \mathrm{L}$ of PI stain was added and mixed at $4^{\circ} \mathrm{C}$ for $30 \mathrm{~min}$, protected from light. The cells were detected by FACScanto 6 flow cytometer and the percentage of cells in G0/G1, S and G2/M phases of the cell cycle $(\%)$ was calculated.

\subsubsection{Osteogenic and lipogenic differentiation induction assay}

The hADSCs of P4 were inoculated into gelatinized 6-well plates. When the 
cells were confluent to $60-70 \%$, the osteogenic differentiation induction medium was changed every 3 days, and after 2-4 weeks of induction, alizarin red staining was performed; When the cells were confluent to $100 \%$, they were replaced with lipogenic differentiation induction medium solution $\mathrm{A}$ and after 3 days with lipogenic differentiation induction medium solution B. After $24 \mathrm{~h}$, solution B was aspirated and solution A was added, and solution A and B were alternately induced 3-5 times, and finally the culture was maintained with solution B for 4-7 days until the lipid droplets became large and round enough for oil red $\mathrm{O}$ staining. After induction of differentiation staining by both methods, 10 randomly selected fields of view were photographed under high magnification using uniform photographic conditions. The area of cells stained with red calcified nodules (osteogenic differentiation) or red lipid droplets (lipogenic differentiation) and the area of all cells in the whole area photographed were measured using Image $\mathrm{J}$ software, and the ratio of the area of differentiated cells to the total area was calculated as the result and expressed as a percentage (\%).

\subsubsection{Reactive oxygen species (ROS) and JC-1 staining assay}

The hADSCs of P4 were stained with JC-1 dye and ROS probe to determine the mitochondrial membrane potential and intracellular ROS content, respectively. Staining was performed according to the instructions of the ROS Fluorescent Probes-DHE and JC-1 kits. Diluted ROS probe solution $(1 \mu \mathrm{M})$ and JC-1 dye $(10 \mu \mathrm{g} / \mathrm{ml})$ were added to the cell well plates and incubated in an incubator at $37^{\circ} \mathrm{C}$ for 10-30min protected from light. After being washed twice with D-PBS, they were observed under a fluorescent microscope and photographed. Cells with weak mitochondrial activity stained red for ROS, while cells stained with JC-1 went from red to green fluorescence, indicating a decrease in mitochondrial membrane potential.

\subsubsection{Statistical Analysis}

Data were reported as mean and standard error, and SPSS 22.0 and Data were expressed as mean and standard error, and data were analyzed using IBM SPSS Statistics 22.0 (IBM, USA) and plotted using Adobe Photoshop Cs5 (Adobe Systems Inc, USA) and GraphPad prism 7 (GraphPad Software, USA). Differences and significance were verified by one-way ANOVA. A p value $<0.05$ was considered as statistically significant.

\section{Results}




\subsection{Cell morphology of hADSCs}

The cell morphology of hADSCs was observed under different temperature gradient treatments, and no morphological differences were observed in hADSCs on the $1^{\text {st }}$ day (Fig 1). When the cells grew to the $3^{\text {rd }}$ day, the cells in the $37^{\circ} \mathrm{C}$ group showed a gradual swirling growth trend, while the cells in the $39^{\circ} \mathrm{C}$ group showed a scattered growth. vacuole-like changes were seen in the cytoplasm of the cells in the $40^{\circ} \mathrm{C}$ group.

$37^{\circ} \mathrm{C}$

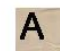

day1

day3

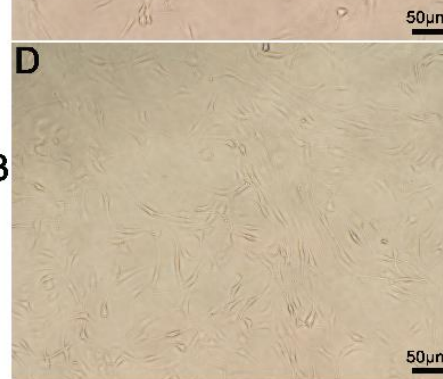

$39^{\circ} \mathrm{C}$

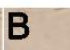

oum
E

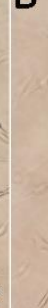

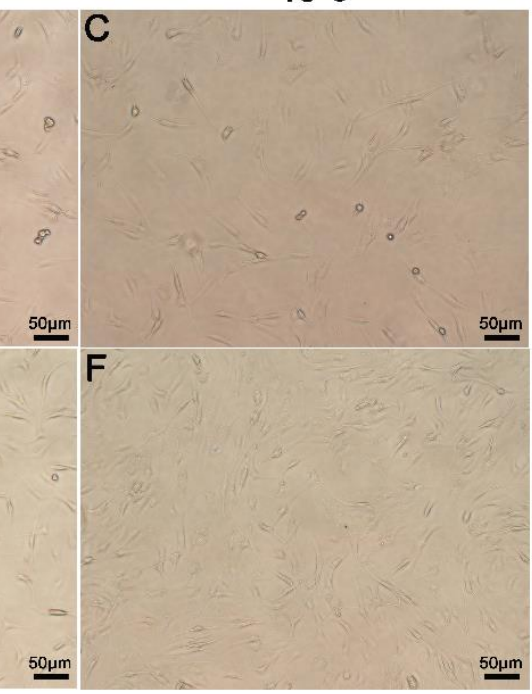

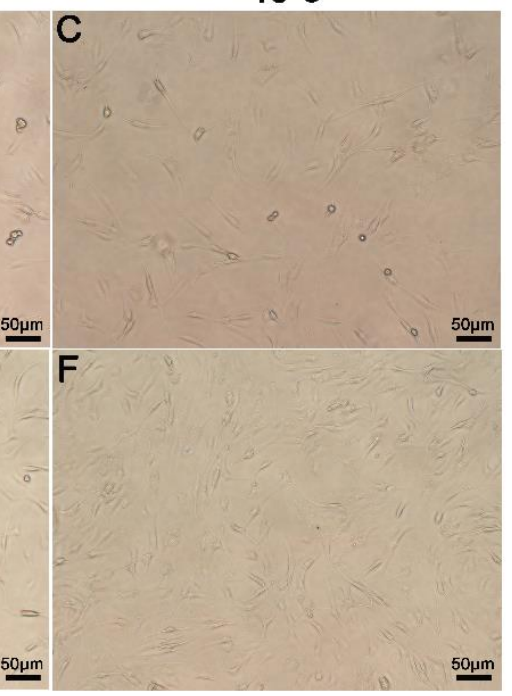

$40^{\circ} \mathrm{C}$

Fig1. Cell morphological characteristics of hADSCs in different temperature treatment groups. A-C were the cell morphology of the $1^{\text {st }}$ day in the $37^{\circ} \mathrm{C}, 39^{\circ} \mathrm{C}$ and $40^{\circ} \mathrm{C}$ groups, respectively, and D-F were the cell morphology of the $3^{\text {rd }}$ day in each group.

\subsection{Cell surface markers of hADSCs}

To understood whether the phenotype of hADSCs changed after different temperature exposures, we performed flow cytometric assays on cell surface markers of hADSCs treated at $37^{\circ} \mathrm{C}, 39^{\circ} \mathrm{C}$ and $40^{\circ} \mathrm{C}$ groups, respectively. The results showed that CD90, CD73 and CD105 expression rates were above 90\%, while CD34 and CD45 expression rates were <3\% (Fig 2). Statistical analysis showed that CD90 and CD73 were not statistically different between groups on the $1^{\text {st }}$ day and the $3^{\text {rd }}$ day, CD105 expression increased with increasing temperature on the $3^{\text {rd }}$ day $(\mathrm{P}<0.5)$, and CD34 and CD45 expression decreased with increasing temperature on the $3^{\text {rd }}$ day $(\mathrm{P}<0.05)$. 

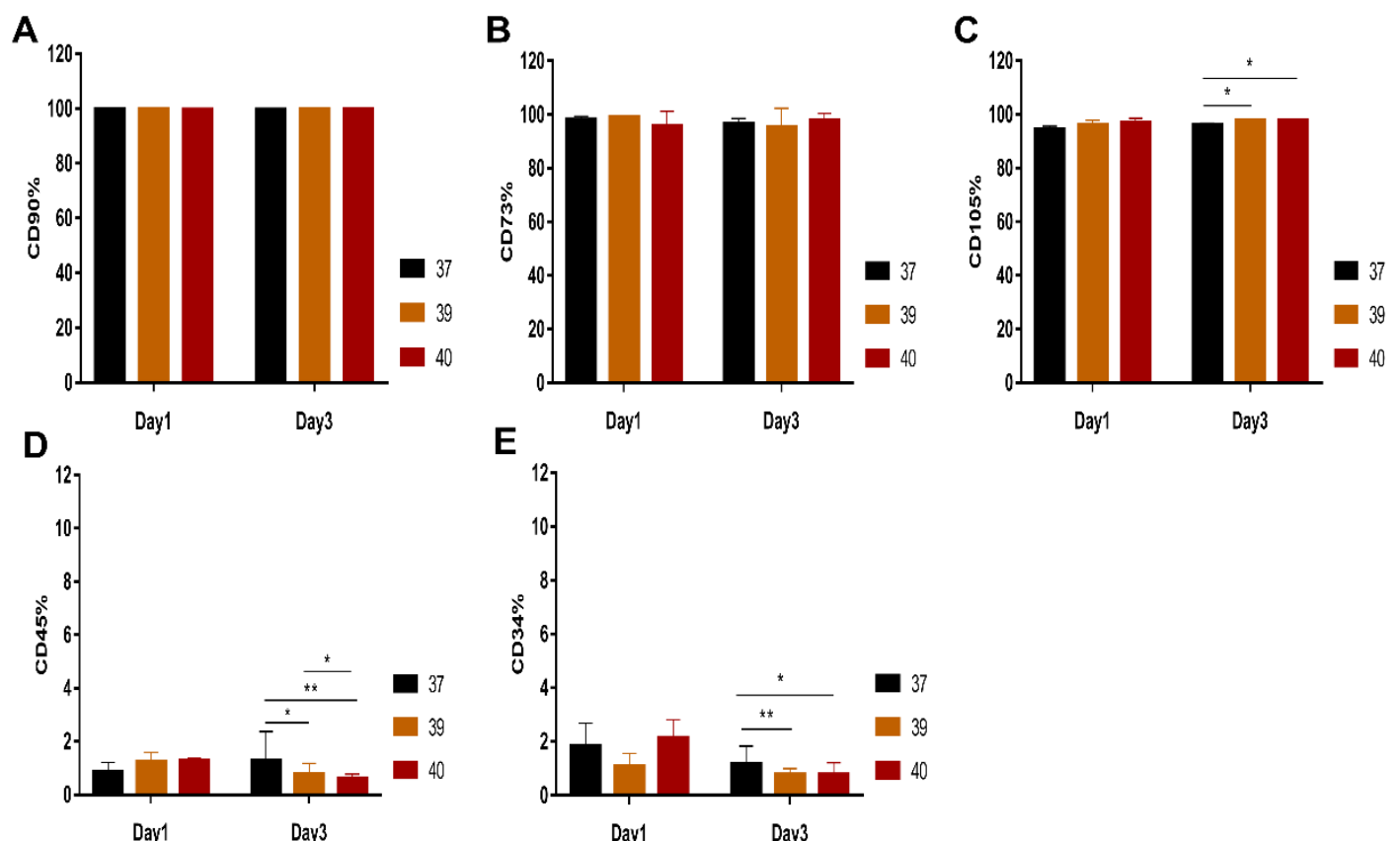

Day1

Fig2. Flow cytometry analysis of cell surface markers. hADSCs were positive for CD90, CD73, CD10 expression and negative for CD45 and CD34 expression. A-E indicates the percentage of each marker on the $1^{\text {st }}$ day and the $3^{\text {rd }}$ day after hADSCs were treated with different temperature groups, respectively. Values bar were expressed as mean $\pm \mathrm{SEM}, * \mathrm{P}<0.05, * * \mathrm{P}<0.01$.

\subsection{Cell proliferation of hADSCs}

This experiment used CCK-8 assay to analyze the proliferation viability of hADSCs at different temperatures $\left(37^{\circ} \mathrm{C}, 39^{\circ} \mathrm{C}\right.$ and $40^{\circ} \mathrm{C}$ group) by measuring the OD values of the cells. The results were shown in Figure 3: on the $1^{\text {st }}$ day, hADSCs were significantly increased in the $39^{\circ} \mathrm{C}$ group compared with the other two groups $(\mathrm{P}<0.05)$; on the $3^{\text {rd }}$ day, there was no statistical difference between the three groups.

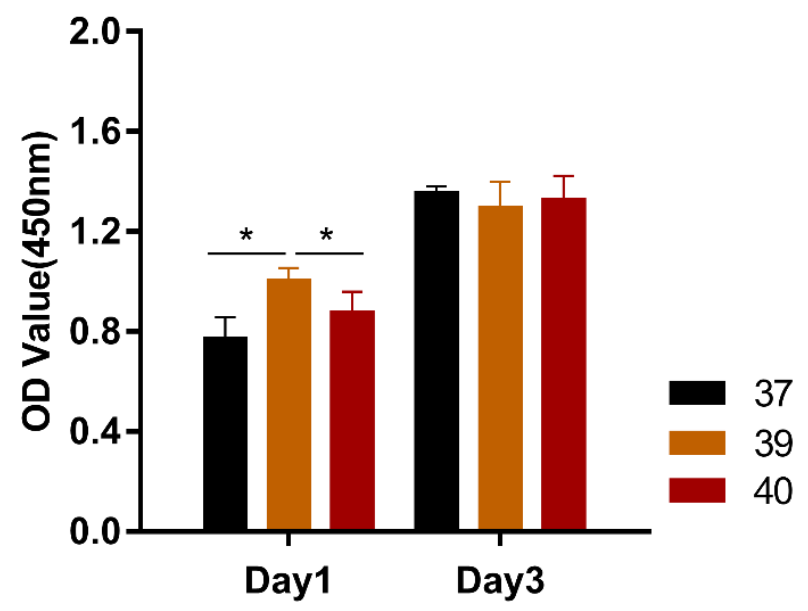

Fig3. Comparison of different temperature treatment groups on the proliferation of hADSCs. 
Values bar were expressed as mean $\pm \mathrm{SEM}, * \mathrm{P}<0.05$.

\subsection{Cell cycle variation in hADSCs}

The cell cycle of hADSCs in each group was measured by flow cytometry, and the results showed (Fig 4) that at the $1^{\text {st }}$ day, the proportion of cells in G1 phase was greater in the $37^{\circ} \mathrm{C}$ group $(50.01 \% \pm 0.40 \%)$ than in the $39^{\circ} \mathrm{C}$ group $(48.53 \% \pm$ $0.96 \%)$ and less than in the $40^{\circ} \mathrm{C}$ group $(54.71 \% \pm 0.61 \%)$, and the difference was statistically significant; the proportion of cells in G2 phase was highest in the $39^{\circ} \mathrm{C}$ group, which was significantly different from the $40^{\circ} \mathrm{C}$ group; the proportion of S-phase of cells in both the $37^{\circ} \mathrm{C}$ and $39^{\circ} \mathrm{C}$ groups was greater than that in the $40^{\circ} \mathrm{C}$ group. At the $3^{\text {rd }}$ day, the G1 phase of all three groups increased compared with the $1^{\text {st }}$ day, and the proportion of G1 phase of cells gradually decreased with the increase of temperature, while the proportion of G2 and S phases of cells gradually increased.

A

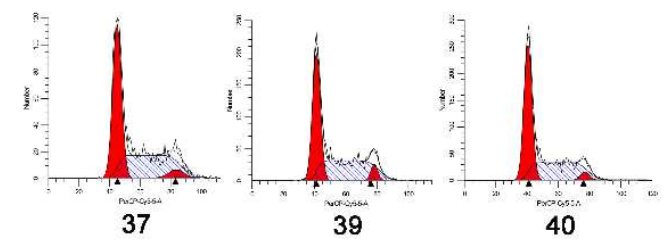

C

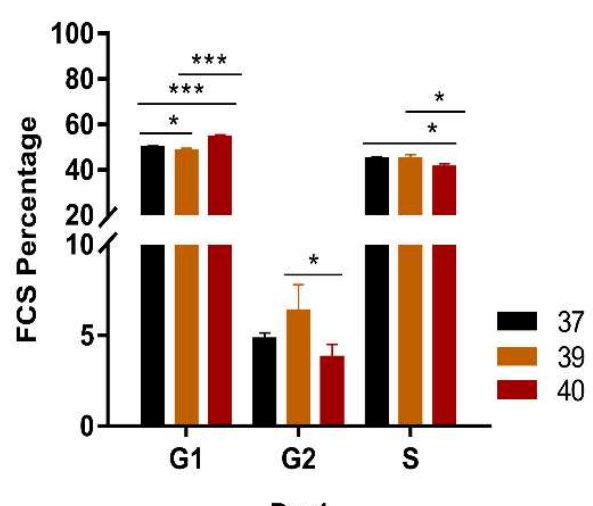

B

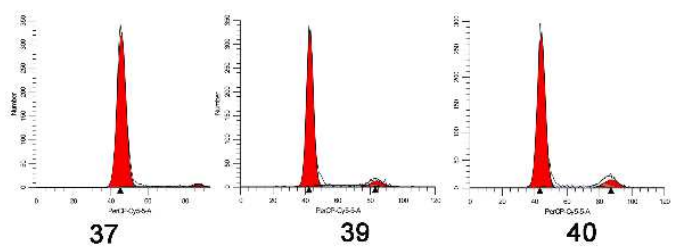

D

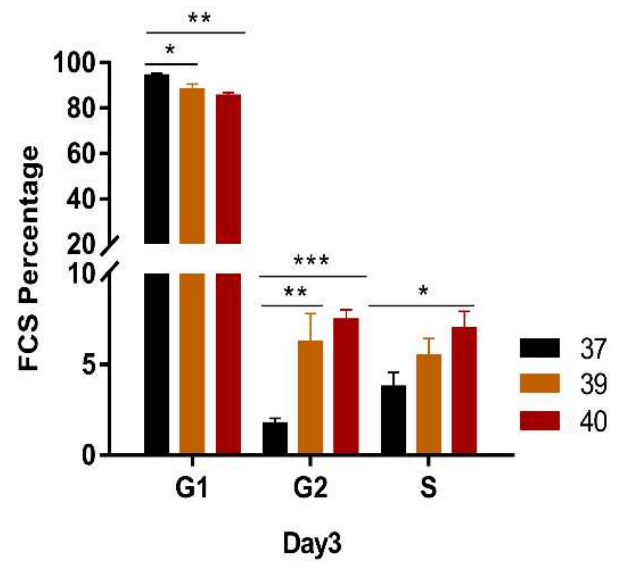

Fig4. Flow cytometry analysis of cell cycle. A and B represent scatter plots of the cell cycle of hADSCs on the $1^{\text {st }}$ day and the $3^{\text {rd }}$ day after treatment with different temperature groups, respectively. C and D represent histograms of the proportion of each phase of the cell cycle of hADSCs on the $1^{\text {st }}$ day and the $3^{\text {rd }}$ day after treatment with different temperature groups, respectively. Values bar were expressed as mean $\pm \mathrm{SEM}, * \mathrm{P}<0.05, * * \mathrm{P}<0.01, * * * \mathrm{P}<0.001$.

\subsection{Osteogenic and lipogenic differentiation ability of hADSCs}

After osteogenesis-induced differentiation of hADSCs from three different temperature-stimulated groups, the formation of red calcified nodules was detected by alizarin red staining, and microscopic observation showed that all three groups of cells 
could form red calcified nodules to different degrees, and the rate of cellular calcium nodule formation increased with the increase of temperature (Fig5.A-F); as shown in the bar graph (Fig5.G), the trend of cellular calcium nodule formation was $37^{\circ} \mathrm{C}$ group $(18.71 \pm 1.73)<39{ }^{\circ} \mathrm{C}$ group $(91.03 \pm 8.03)<40{ }^{\circ} \mathrm{C}$ group $(103.58 \pm 7.32)(\mathrm{P}<0.01)$. After lipogenesis-induced differentiation of hADSCs from three different temperature stimulation groups, they were stained with oil red O. Microscopic observation showed that all three groups of cells showed lipid droplets of different sizes (Fig6.A-F), the saturation of cellular lipid droplets decreased with increasing temperature, and the size of cellular lipid droplets showed $37^{\circ} \mathrm{C}$ group $(32.90 \pm 4.32)>39^{\circ} \mathrm{C}$ group $(28.19 \pm 1.32)>40^{\circ} \mathrm{C}$ group ( $\left.26.69 \pm 2.55\right)$, with a statistical difference between the $37^{\circ} \mathrm{C}$ and $40^{\circ} \mathrm{C}$ groups.
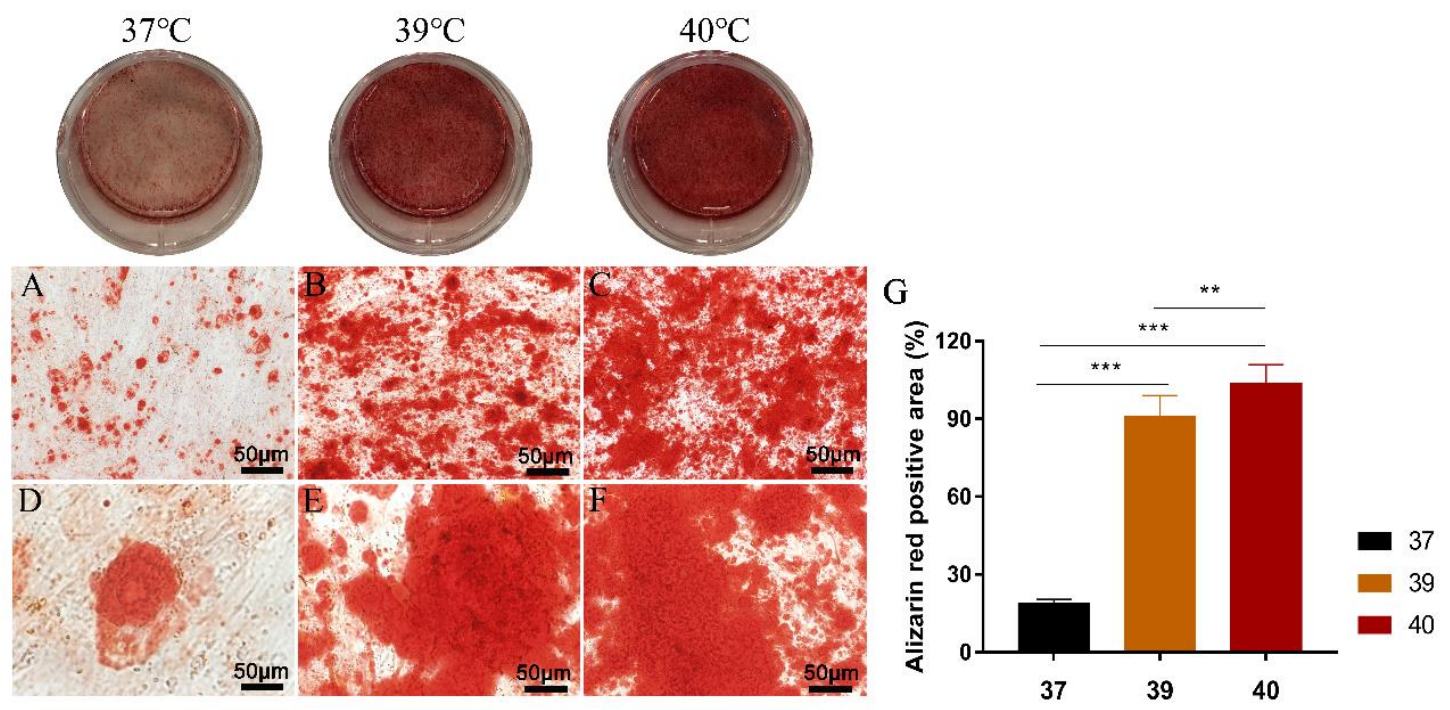

Fig5. Osteogenic differentiation potential of hADSCs after different temperature treatment. A-C were the results of alizarin red staining in the $37{ }^{\circ} \mathrm{C}$ group, $39{ }^{\circ} \mathrm{C}$ group and $40{ }^{\circ} \mathrm{C}$ group observed at low magnification, while D-F represent their results at high magnification, respectively. G was the quantification of alizarin red staining score. Values bar were expressed as mean $\pm \mathrm{SEM}$, **P < $0.01, * * * \mathrm{P}<0.001$. 

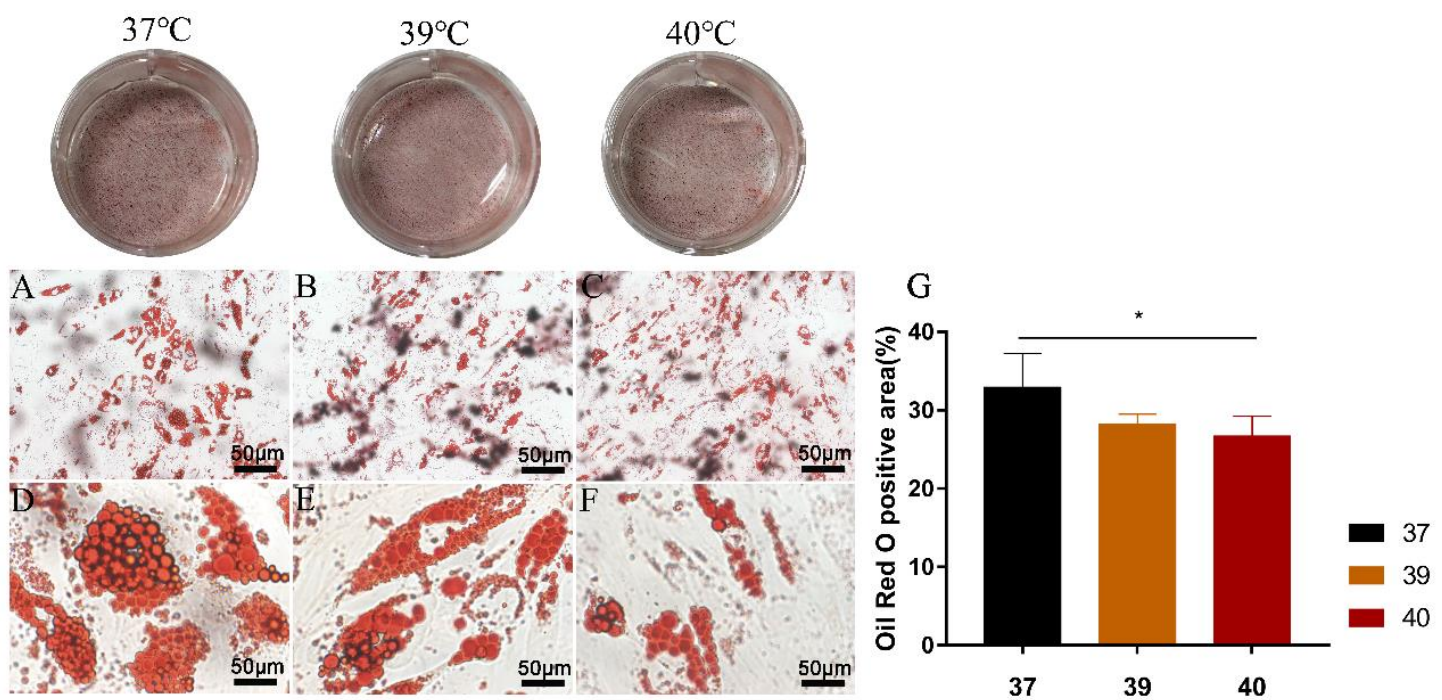

Fig6. Lipogenic differentiation potential of hADSCs after different temperature treatment. A-C were the results of oil-red $\mathrm{O}$ staining observed at low magnification for the $37{ }^{\circ} \mathrm{C}$ group, $39{ }^{\circ} \mathrm{C}$ group and $40{ }^{\circ} \mathrm{C}$ group, while D-F represent their results at high magnification, respectively. G was the quantification of oil-red $\mathrm{O}$ staining scores. Values bar were expressed as mean $\pm \mathrm{SEM}, * \mathrm{P}<$ 0.05 .

\subsection{Results of ROS and JC-1 assay}

The ROS content of hADSCs on the $1^{\text {st }}$ day and the $3^{\text {rd }}$ day after different temperature treatments in the three groups was measured by ROS probes. As shown in Fig 7 , at the $1^{\text {st }}$ day, the ROS fluorescence intensity was weak and not significantly different between the $37^{\circ} \mathrm{C}$ and $39^{\circ} \mathrm{C}$ groups, while the ROS fluorescence intensity was significantly enhanced in the high temperature $\left(40^{\circ} \mathrm{C}\right)$ group; at the $3^{\text {rd }}$ day, the fluorescence was still weak in the $37^{\circ} \mathrm{C}$ group, while the ROS fluorescence expression intensity was significantly enhanced in the $39^{\circ} \mathrm{C}$ and $40^{\circ} \mathrm{C}$ groups. The results of mitochondrial membrane potential measurement using JC-1 dye were shown in Figure 8 , which showed that the trend of mitochondrial membrane potential change was a gradual increase in membrane potential depolarization with the increase in ambient temperature. The effect of JC-1 membrane potential sensitive dye on mitochondrial depolarization was observed by fluorescence microscopy, with high potentials characterized by red fluorescence, while low potentials showed green fluorescence. The results showed that the depolarization was more pronounced at higher temperatures. 


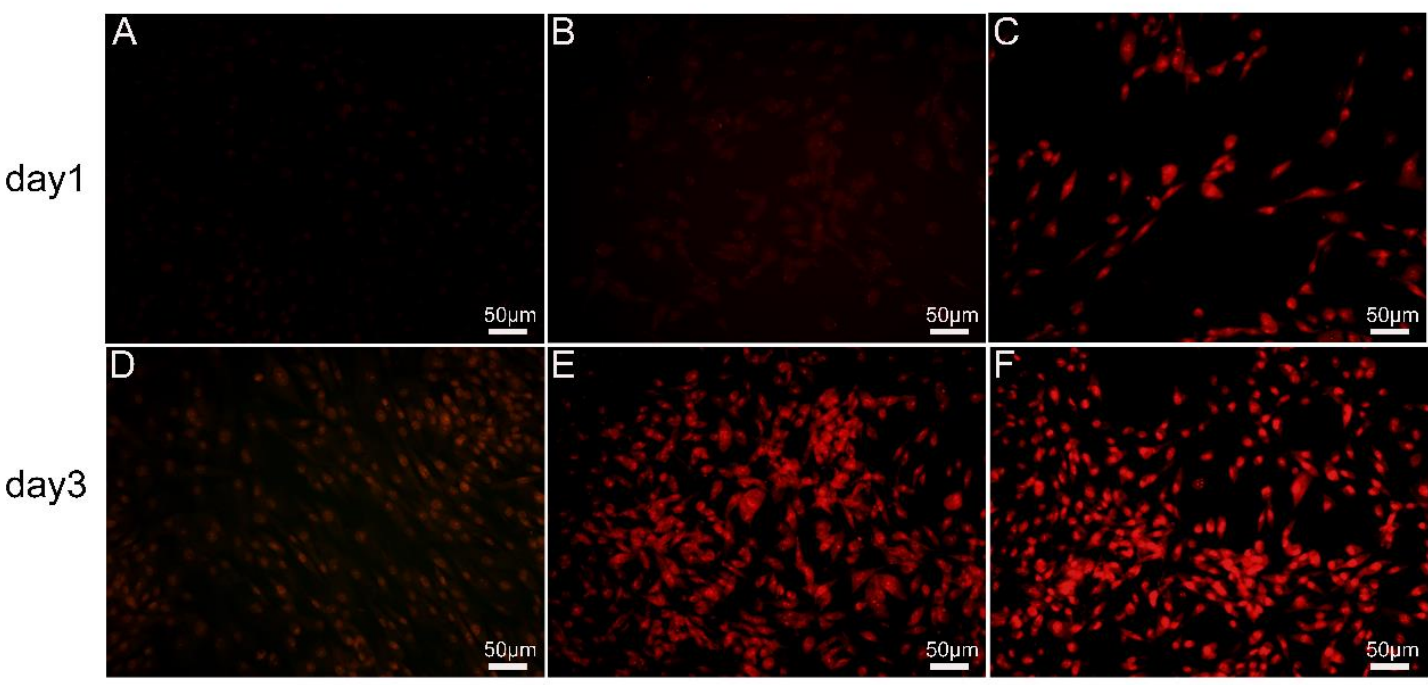

Fig7. Effect of different temperature groups on ROS content of hADSCs. A-C were the expression of ROS of hADSCs at the $1^{\text {st }}$ day; D-F were the expression of ROS of hADSCs at the $3^{\text {rd }}$ day.

$37^{\circ} \mathrm{C}$

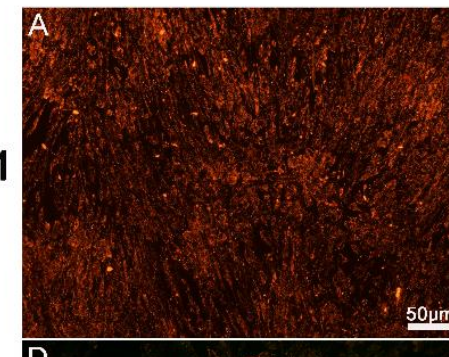

day1

\section{Discussion}

$39^{\circ} \mathrm{C}$

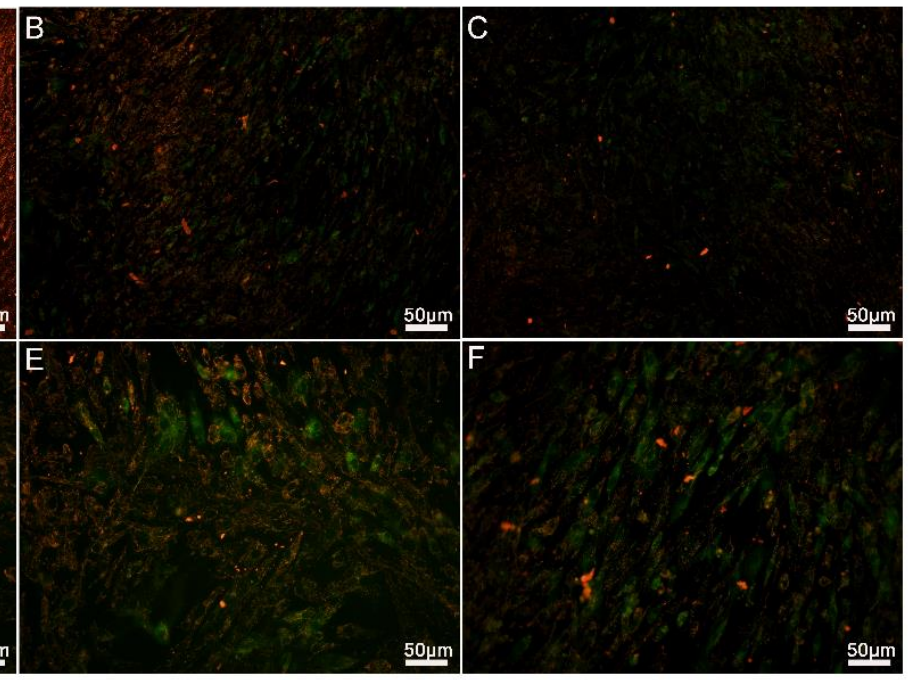

Fig 8. Effect of different temperature groups on mitochondrial depolarization of hADSCs. A-C were the mitochondrial membrane potential changes of hADSCs at the $1^{\text {st }}$ day; D-F were the mitochondrial membrane potential changes of hADSCs at the $3^{\text {rd }}$ day.

Global warming will affect ecosystems as well as human health in multiple ways, and these impacts are expected to rise dramatically with increasing warming, with estimates that the number of people at climate-related risk will increase by hundreds of millions by 2050. However, it remains difficult to predict the human impacts of the complex interplay of mechanisms driven by warming ${ }^{[12]}$. At present, the impact of climate warming on human survival and in animals is still poorly understood. 
Yellow adipose tissue is mainly distributed in subcutaneous tissues, omentum and mesentery, and is the largest energy reservoir in the body. It has the function of storing fat, maintaining and regulating body temperature and participating in fat metabolism ${ }^{[13]}$. There are more reports on how adipose tissue responds to changes in the external environment at low temperatures, but few studies have examined the changes in adipose tissue at high temperatures. In this study, we chose adiposederived human adipose mesenchymal stromal cells as the subject to explore the biological properties of the cells and the changes in oxidative stress under high temperature environment.

Available reports show that positive expression of CD73, CD105 and CD90, and negative expression of CD34 and CD45 are cell surface markers of ADSCs ${ }^{[14,15]}$. To study showed no significant changes from the cell morphology, but subtle differences in their cell surface markers have been observed. At the $3^{\text {rd }}$ day of treatment at different temperatures, the expression of CD105 was increased at $39^{\circ} \mathrm{C}$ and $40^{\circ} \mathrm{C}$ compared to $37^{\circ} \mathrm{C}$, indicating an enhanced chondrogenic capacity ${ }^{[16]}$. In contrast, the expression of CD34 and CD45 decreased and was $<5 \%$, suggesting that the cells still have the characteristics of mesenchymal stromal cells [17]. Analysis of cell proliferation viability showed that at $24 \mathrm{~h}$ after passaging, the proliferation capacity of hADSCs in the $39^{\circ} \mathrm{C}$ environment was significantly higher than the other two groups, and by $72 \mathrm{~h}$, the proliferation capacity of cells in the $37^{\circ} \mathrm{C}$ group was slightly higher than the others, showing that the change in environmental high temperature caused a short-term rapid proliferation of hADSCs, while the opposite proliferation trend occurred by $72 \mathrm{~h}$. Further analysis in terms of cell cycle showed that at the $3^{\text {rd }}$ day of cell growth, the G1 phase (pre-DNA synthesis phase) was reduced and the $\mathrm{S}$ phase (DNA replication phase) and G2 phase (late DNA synthesis phase) were significantly higher in the $39^{\circ} \mathrm{C}$ and $40^{\circ} \mathrm{C}$ environments compared to the $37^{\circ} \mathrm{C}$ group, illustrating that the $\mathrm{S}$ and $\mathrm{G} 2$ phases of the cells were blocked and the cell division ability was reduced, which were consistent with the formation of a gradual decrease in cell proliferation activity at the $3^{\text {rd }}$ day.

The multidirectional differentiation potential of stromal cells is one of the functions of stromal cells measured. This experiment showed that the rate of cellular calcium nodule formation increased with increasing temperature after osteogenesisinduced differentiation of hADSCs in each group, and the saturation of cellular lipid 
313 droplets decreased with increasing temperature after lipogenesis-induced 314 differentiation. This indicates that temperature exerts an important influence on the differentiation of stromal cells. Jang et al ${ }^{[18]}$ compared the differences in indoor and outdoor summer temperatures and humidity in Korea and Southeast Asia to assess changes in skin studies showed that repeated exposure to high temperatures and high humidity increased sebum content and hemoglobin index in human skin. However, repeated temperature differences can relax skin blood vessels and reduce skin elasticity, which can contribute to skin aging. These results were associated with a greater tendency for cells to become chondrogenic and differentiated at elevated temperatures. Kim et al ${ }^{[19]}$ recruited 20 women aged 20 to 40 years. After exposing them to outdoor and indoor environments for 90 minutes, skin assessments were performed on the face (forehead and cheeks) and forearms to determine the degree of skin hydration, sebum secretion, transepidermal water loss, $\mathrm{pH}$, and oil content. The study showed that hot environments produce more sweat, increase hydration, sebum secretion, water loss, and lower skin $\mathrm{pH}$. These results suggest that hot environments produce varying degrees of disruption to human skin and to the function and metabolism of epidermal cells. These results are relatively similar to the tendency of hADSCs to osteogenic differentiation by high-temperature environments.

Proton pumps are present in the inner mitochondrial membrane and can pump matrix protons into the membrane gap. Proton translocation across the membrane allows the mitochondrial membrane gap to accumulate large internal amounts of protons, described as a proton gradient: the mitochondrial membrane gap generates a large positive charge while the mitochondrial matrix generates a large negative charge, resulting in a transmembrane potential $(\Delta \Psi)$ across the inner mitochondrial membrane, referred to as the mitochondrial membrane potential. Normal mitochondrial membrane potential is a prerequisite for maintaining oxidative phosphorylation and ATP production in mitochondria and is necessary for maintaining mitochondrial function, while an important change during apoptosis is the collapse of mitochondrial membrane potential ${ }^{[20]}$. JC-1 is an ideal fluorescent probe widely used to detect mitochondrial membrane electrical $\Delta \Psi \mathrm{m}$ sites. JC-1 dye accumulates within mitochondria in a potential- dependent manner and can be used to detect cellular, tissue or purified mitochondrial membrane potentials. In normal mitochondria, JC-1 aggregates in the mitochondrial matrix to form polymers, which emit intense red fluorescence $(E x=585 \mathrm{~nm}, E m=590 \mathrm{~nm})$; in unhealthy mitochondria, due to a decrease 
or loss of membrane potential, JC-1 can only exist as a monomer in the cytoplasm, producing green fluorescence $(E x=514 \mathrm{~nm}, \mathrm{Em}=529 \mathrm{~nm})$. JC-1 is not only used for qualitative detection, because the change of color can reflect the change of mitochondrial membrane potential very directly, but also for quantitative detection, because the degree of mitochondrial depolarization can be measured by the ratio of red/green fluorescence intensity. From the experimental results, it was observed that the JC-1 staining changed from red to green with the increase of temperature, suggesting that the mitochondrial membrane potential depolarization was serious at high temperature, and the membrane potential decreased and apoptosis occurred in the cells.

ROS is a class of single-electron reduction products of oxygen in the body, which are generated by leaking electrons out of the oxidative respiratory chain and consuming about $2 \%$ of the oxygen before it is passed to the terminal oxidase, including the one-electron reduction product of oxygen, superoxide anion, twoelectron reduction product, hydrogen peroxide, three-electron reduction product, hydroxyl radical, and nitric oxide. ROS production is mainly formed by the high oxygen environment and the high reduced state of the respiratory chain in the mitochondrial transition from state III to state IV, which causes a large number of electrons to leak out and reduce oxygen molecules. Under pathological conditions, the loss of the normal balance between the production and removal of ROS often results in damage to the body by ROS. Oxidative damage mainly includes: first, oxidative damage to nucleic acids, after oxidative damage to the DNA may occur breaks, mutations and changes in thermal stability, which seriously affects the normal transcription and translation of genetic information; second, modification of amino acids, peptide chain breaks, the formation of cross-linked polymers of proteins, changes in conformation and immunogenicity, and other five aspects. Most of the hydroxyl radicals in the organism are produced in the organelles, especially in the mitochondria, causing damage to the mitochondrial membrane and leading to impaired energy metabolism in the cells and the organism ${ }^{[21-22]}$. The present study showed that with increasing temperature, mitochondrial oxidative stress ROS content increases, demonstrating increased production and release of mitochondrial oxides and inhibited activity.

The results of ROS and JC- 1 assays were in line with the mechanistic studies in humans when heat stroke occurs, such as in heat stroke, cells in the body are in a 
hypoxic state, ATP is reduced, $\mathrm{Ca}^{2+}$ concentration is increased, followed by elevated ROS and oxidative stress, leading to apoptosis, tissue necrosis and autophagy, and eventually multi-organ dysfunction and individual death ${ }^{[23-24]}$. Interestingly, the results in this experiment showed that ROS was elevated and mitochondrial activity was reduced when hADSCs cells were in a high temperature environment; the cell membrane depolarization was severe during JC-1 staining, which may also lead to dysregulation of $\mathrm{Ca}^{2+}$ flow. It may further explain that when people are under high temperature all the time, the growth of hADSCs cells is restricted and cellular oxidative stress is severe, and then apoptosis or cell death occurs, resulting in the loss of the ability of adipocytes to maintain body temperature, and the final trend is the loss of organismal function and death.

ADSCs are widely distributed in vivo, including subcutaneous tissues. The changes in cellular activity and function of hADSCs induced by high temperature are closely related to explain the aging of epidermal cells, dysregulation of lipid metabolism and maintenance of body temperature. Therefore, we hypothesize that humans and animals growing near the equator or in hot regions are affected by high temperatures, which may cause aging of surface cells such as skin in mild cases and impairment of metabolism of cells, tissues and organs in the body in severe cases, thus causing various diseases.

In conclusion, hADSCs grown under high temperature conditions have restricted growth activity, blocked $\mathrm{S}$ and $\mathrm{G} 2$ phases resulting in reduced cell division, enhanced osteogenic differentiation, impaired mitochondrial activity, and severe cell depolarization. These phenomena are directly related to skin aging, apoptosis and heat stroke diseases caused by outdoor exposure to sunlight in summer as reported by previous researchers. These results may provide a further explanation for global warming to localize high temperatures, causing the cause of species extinction.

\section{Abbreviations}

hADSCs: human adipose-derived mesenchymal stromal cells; ROS: Reactive oxygen species.

\section{Acknowledgments}

The authors thank all students and technicians in the laboratory for their cooperation. 


\begin{tabular}{|c|c|}
\hline Yuanhui Gao & performed experiments, provided funding supported \\
\hline Hui Cao & performed experiments \\
\hline Shunlan Wang & performed experiments \\
\hline Linlin Zheng & performed experiments \\
\hline Haowei He & performed experiments \\
\hline Zhenyu Nie & helped with the experiments \\
\hline Mei Chen & helped with the experiments \\
\hline Rong Jiang & discussed with clinical disease \\
\hline Denggao Huang & $\begin{array}{l}\text { provided funding supported, provided the expert opinion in } \\
\text { temperature design, co-wrote and finalized the manuscript text, } \\
\text { monitored the research design and progress }\end{array}$ \\
\hline Shufang Zhang & $\begin{array}{l}\text { provided funding supported, monitored the research design and } \\
\text { progress }\end{array}$ \\
\hline
\end{tabular}

\section{Funding}

This research was supported by the Hainan Provincial Natural Science Foundation of

\section{Availability of data and materials}

The data that support the findings of this study are available from the corresponding author upon request.

Ethics approval and consent to participate

Not applicable.

\section{Consent for publication}

429 Not applicable.

\section{Competing interests}

432 The authors declare that they have no competing interests.

\section{Author information}


${ }^{1}$ Department of Central Laboratory, Afliated Haikou Hospital of Xiangya Medical College, Central South University, Haikou 570208, Hainan, China

${ }^{2}$ Department of General Surgery, 900th Hospital of the Joint Logistics Team/Clinical Institute of Fuzhou General Hospital, Fujian Medical University/Dongfang Hospital Affiliated of Xiamen University, Fuzhou 350025, Fujian, China

\section{References}

[1] Jeremy M Cohen, Erin L Sauer, Olivia Santiago, Samuel Spencer, Jason R Rohr. Divergent impacts of warming weather on wildlife disease risk across climates. Science. 2020, 370(6519): eabb1702.

[2] Christopher H Trisos, Cory Merow, Alex L Pigot. The projected timing of abrupt ecological disruption from climate change. Nature. 2020, 580(7804): 496- 501.

[3] Ken Kobayashi, Yusaku Tsugami, Kota Matsunaga, Takahiro Suzuki, Takahiro Nishimura. Moderate High Temperature Condition Induces the Lactation Capacity of Mammary Epithelial Cells Through Control of STAT3 and STAT5 Signaling. J Mammary Gland Biol Neoplasia. 2018 Jun;23(1-2):75-88.

[4] Torlińska T, Banach R, Paluszak J, Gryczka-Dziadecka A. Hyperthermia effect on lipolytic processes in rat blood and adipose tissue. Acta Physiol Pol. 1987, 38(4):361-6.

[6] Qi Zhao, Yuming Guo, Tingting Ye, et al. Global, regional, and national burden of mortality asso ciated with non-optimal ambient temperatures from 2000 to 2019: a three-stage modelling study. Lancet Planet Health. 2021, 5(7): e415-e425.

[7] A. Bouchama and J. P. Knochel. Heat stroke. N Engl J Med. 2002, 346(25): 1978-1988.

[8] Lu Kuo-Cheng, Wang Jia-Yi, Lin Shih-Hua, et al. Role of circulating cytokines and chemokines in exertional heatstroke. Critical care medicine, 2004, 32(2): 399-403.

[9] María E Frigolet, Ruth Gutiérrez-Aguilar. The colors of adipose tissue. Gac Med Mex. 2020; 156(2):142-149.

[10] Ziad Obermeyer, Jasmeet K Samra, Sendhil Mullainathan. Individual differences in normal body temperature: longitudinal big data analysis of patient records. BMJ. 2017, 359: j5468.

[11] Michael Gurven, Thomas S Kraft, Sarah Alami, Juan Copajira Adrian, et al. Rapidly declining body temperature in a tropical human population. Sci Adv. 2020, 6(44): eabc6599.

[12] Chi Xu, Timothy A Kohler, Timothy M Lenton, Jens-Christian Svenning, Marten Scheffer. Future of the human climate niche. Proc Natl Acad Sci U S A. 2020,117(21):11350-11355.

[13] Symonds ME, Aldiss P, Dellschaft N, Law J, Fainberg HP, Pope M, Sacks H, Budge H. Brown adipose tissue development and function and its impact on reproduction. $\mathrm{J}$ Endocrinol. 2018, 238(1): R53-R62.

[14] Ludovic Zimmerlin, Vera S Donnenberg, J Peter Rubin, Albert D Donnenberg. Mesenchymal 
markers on human adipose stem/progenitor cells. Cytometry A. 2013, 83(1):134-40.

[15] Alexander Mildmay-White, Wasim Khan. Cell Surface Markers on Adipose- Derived Stem Cells: A Systematic Review. Curr Stem Cell Res Ther. 2017, 12(6): 484-492.

[16] Ting Jiang, Wei Liu, Xiaojie Lv, Hengyun Sun, Lu Zhang, Yu Liu, Wen Jie Zhang, Yilin Cao, Guangdong Zhou. Biomaterials. 2010, 31(13):3564-71.

[17] Imran Ullah, Raghavendra Baregundi Subbarao, Gyu Jin Rho. Human mesenchymal stem cells - current trends and future prospective. Biosci Rep. 2015,35(2): e00191.

[18] Sue Im Jang, Myeongryeol Lee, Yu Chul Jung, Min Kyung Jeong, Ja Hyun Ryu , Beom Joon Kim, Byung-Fhy Suh, Eunjoo Kim. Skin characteristics following repeated exposure to simulated outdoor and indoor summer temperatures in South Korea and Southeast Asia. Int J Cosmet Sci. 2021,17.

[19]S Kim, J W Park, Y Yeon, J Y Han, E Kim. Influence of exposure to summer environments on skin properties. J Eur Acad Dermatol Venereol. 2019, 33(11): 2192- 2196.

[20] Tatiana K Rostovtseva, Wenzhi Tan, Marco Colombini. On the role of VDAC in apoptosis: fact and fiction. J Bioenerg Biomembr. 2005, 37(3):129-42.

[21] Jakubczyk K, Dec K, Kałduńska J, Kawczuga D, Kochman J, Janda K. Reactive oxygen species - sources, functions, oxidative damage. Pol Merkur Lekarski. 2020, 48(284):124-127.

[22] Tan DQ, Suda T. Reactive Oxygen Species and Mitochondrial Homeostasis as Regulators of Stem Cell Fate and Function. Antioxid Redox Signal. 2018, 29(2):149-168.

[23] Sheng-Hsien Chen, Mao-Tsun Lin, Ching-Ping Chang. Ischemic and oxidative damage to the hypothalamus may be responsible for heat stroke. Curr Neuropharmacol. 2013, 11(2):129-40.

[24] Hsin-Hsueh Shen, Yu-Shiuan Tseng, Ni-Chun Kuo, et al. Alpha-Lipoic Acid Protects Cardiomyocytes against Heat Stroke-Induced Apoptosis and Inflammatory Responses Associated with the Induction of Hsp70 and Activation of Autophagy. Mediators Inflamm. 2019, 2019: 8187529. 